\title{
Information collection system of agricultural traceability based on things
}

\author{
Li Tingting ${ }^{1}$, Li Bo ${ }^{2}$, Huang Dechang ${ }^{2}$ \\ ${ }^{1}$ School of Software Engineering, East China Jiaotong University, Nanchang, 330013, PR China \\ ${ }^{2}$ School of Information Engineering, East China Jiaotong University, Nanchang, 330013, PR China
}

Email: libo_jiaoda@126.com

Keywords: Things agriculture; Elman prediction model; Stratified case-based reasoning; Network optimization.

\begin{abstract}
Things agriculture is an important development direction of networking applications, networking technology in the field of agriculture for the development of agriculture has brought immeasurable impetus and prospects immeasurable. In agriculture things, how can the production process the data collected for processing, analysis and display, making it better for agricultural services is needed to solve critical issues. Data processing agricultural Things is located in agricultural information networking application layer through data processing, integration and application to the development of scientific management decisions, to achieve the agricultural production process control.
\end{abstract}

\section{Introduction}

Things application is very popular broad, indeed omnipresent. Defense, military, precision agriculture, digital oilfield, smart home, personal health are filled with things of the shadow [1]. Meanwhile, the human way of life has also undergone a change, "feel" up to "sense", "sense" up to "control." It is foreseeable that things will bring structural adjustment and industrial upgrading of traditional industrial sectors throughout the country, and will promote the adjustment of economic structure [2]. Things definition can be further elaborated as follows: Things are in such a network, which is in accordance with the agreed protocol, through information sensing equipment, such as: radio frequency identification (RFID) devices, infrared sensors, global positioning systems, laser scanners, etc. the articles connected to the Internet for communication and information exchange, to achieve intelligent identification, positioning, tracking, monitoring and management [3, 4].

Things existing agricultural research focused on the use of agricultural resources, agro-ecological environment monitoring, agro-ecological sophisticated management, safety of agricultural products traceability, etc., the key technologies in agriculture three-tier model where things are covered, and expand through aspect perception, transmission, processing and other agricultural information. While agricultural information processing technology, including agricultural forecasting warning technology, intelligent control of agricultural technology, agricultural intelligent decision technology, agricultural technology diagnostic reasoning, visual processing agricultural technology [5-7].

This paper studies the agricultural IOT data processing services system. Then he describes the current profiles have some types of agricultural data processing services system. On this basis, through in-depth analysis of the agricultural production process, user demand for agricultural data processing services for data processing agricultural Things summarize key technologies. Agricultural production control workflow access control technology. After application status and access control system can be customized in the application of the problems set forth proposed access control model attributes and task-based. After a detailed description of its architecture and mathematical proofs later, given its operational framework. Finally, an example of a specific project by the actual needs, verify its feasibility. 


\section{Agriculture Things Data Processing and Services System}

Traceability System. Traceability system is also called traceability system, which is in the process of the product throughout the supply of various product-related information will be recorded and stored, in order to ensure product quality system. When can the purpose of traceability system to problems in product quality, can quickly and efficiently query to link problems to recall the product when necessary, and implement appropriate punitive measures, thereby to improve to ensure product quality $[8,9]$.

SOA based on the quality of agricultural products traceability system supply chain business processes and functions in the form of services provided. By coding process logic and the underlying application isolation between applications call each other through the service. The overall architecture of the system divided into four layers: user interaction layer, business logic, the Service Provider, data application layer (Figure 1).

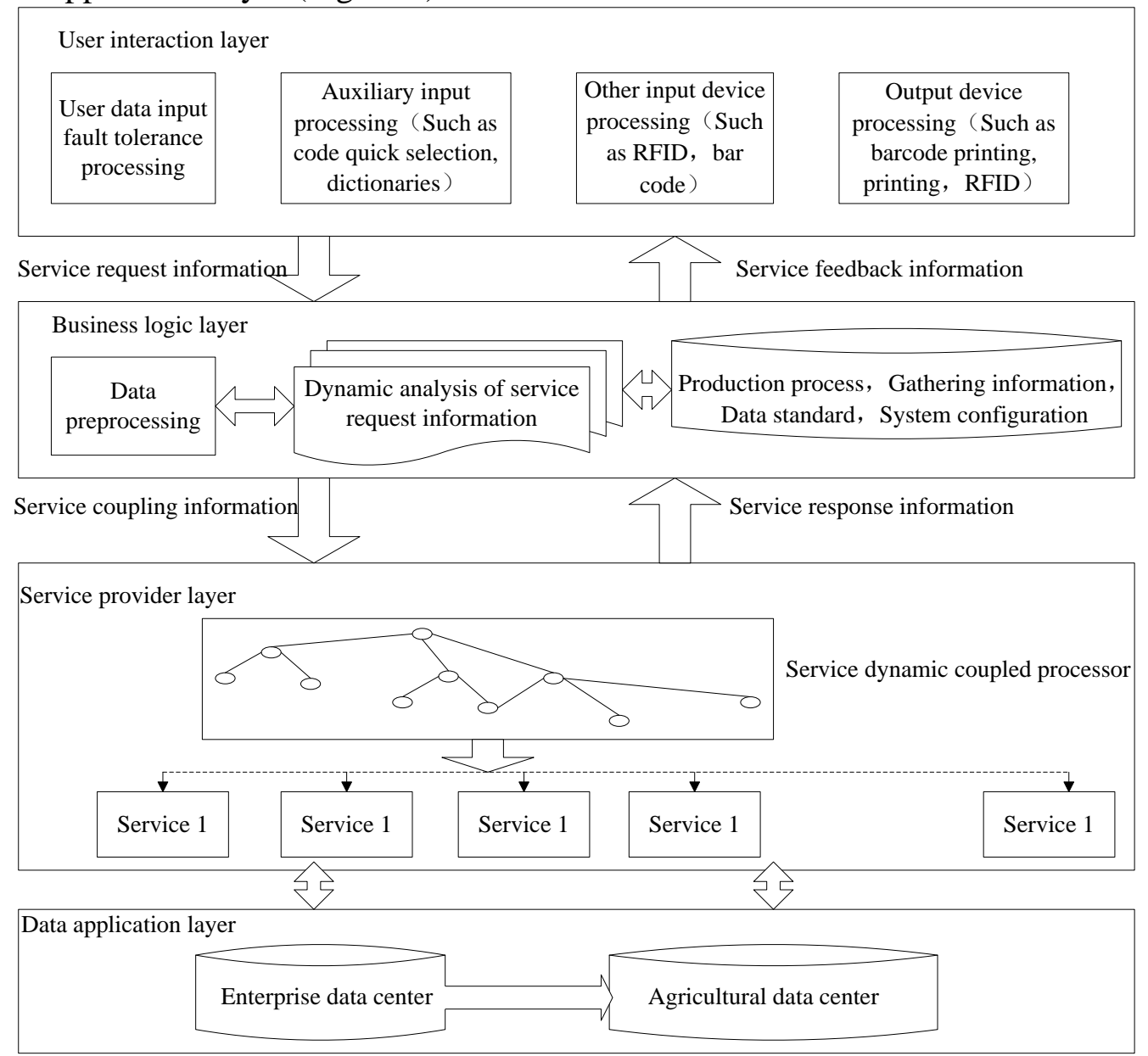

Fig. 1. Agricultural product quality traceability system architecture based on SOA

Client interface invoked by user input parameters to invoke the business logic layer of services; analysis of customer needs, and user needs corresponding service call interface interface to the user. Service calls are completely transparent to the user only needs to provide the appropriate parameters based on the interface and functional description can complete the call, the service execution is complete, automatically returns the results [10].

Data processing needs. Planting and breeding class production process, all kinds of unexpected situations often arise, these emergency situations including both emergency situations exist agricultural growth process itself, but also a variety of emergency situations occurring in the growth environment. This requires the environment of the production process and production data in real-time information analysis and forecasting of possible problems in real-time early warning in order to quickly solve the agricultural production process of all kinds of unexpected problems, to reduce the loss of agricultural production. 
In the process of agricultural production, agricultural production for all kinds of problems arise, for less experienced staff who will have to obtain a solution, is often more difficult. But the update and increase their knowledge of not just rely on input agriculture expert knowledge, which requires data collected through the past and predict the results of extracting knowledge to increase additional knowledge content. Figure 2 shows a typical application needs agricultural data processing services are facing.

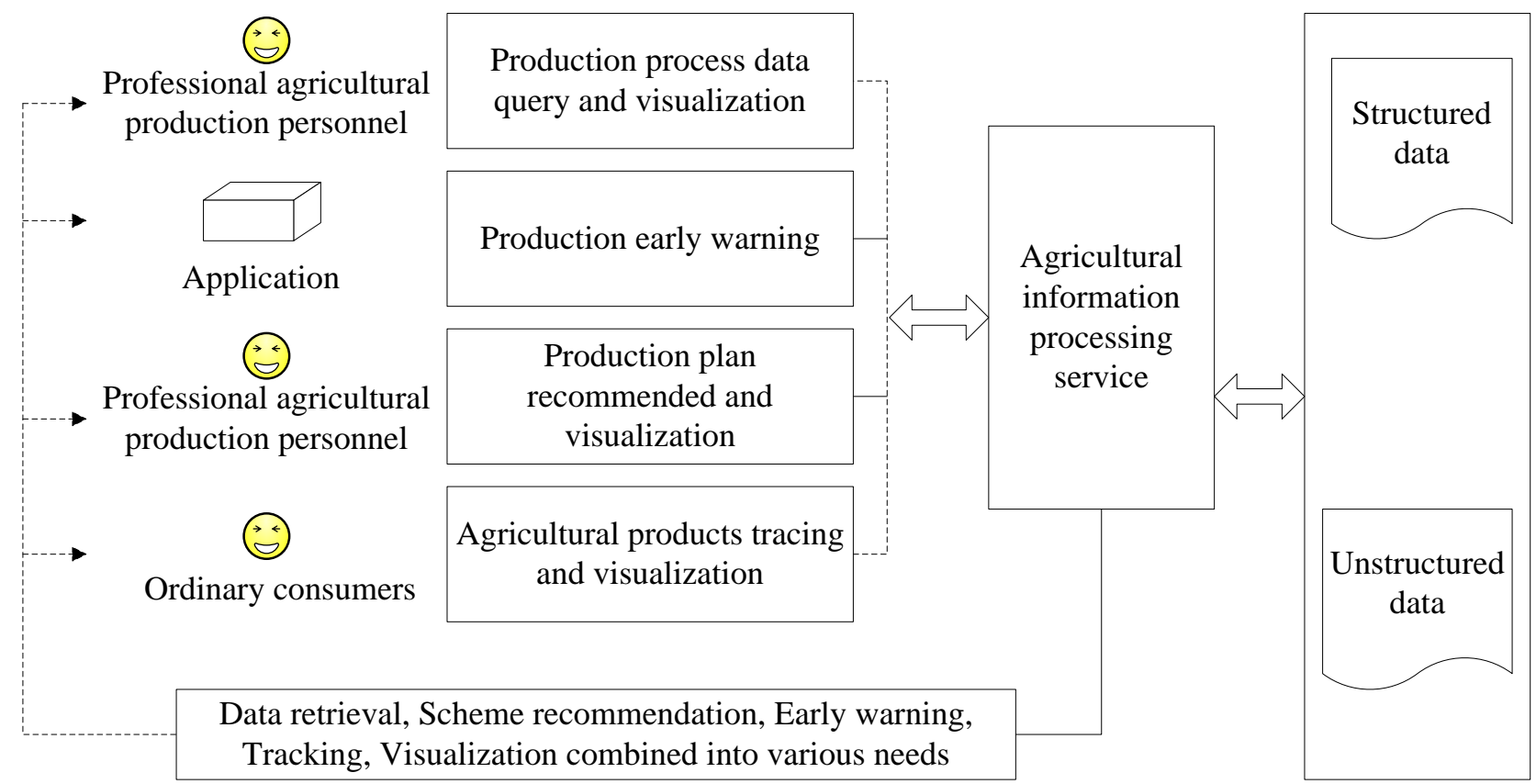

Figure 2. Applications for the agricultural production process data processing services

\section{Experimental results}

Application example model. System specifications according to the appropriate category of agricultural HACCP and GAP system in the critical control point to build knowledge processing and knowledge representation framework. Cotton category, for example, based on class - represents the policy was shown in Figure 3 production rules knowledge - framework.

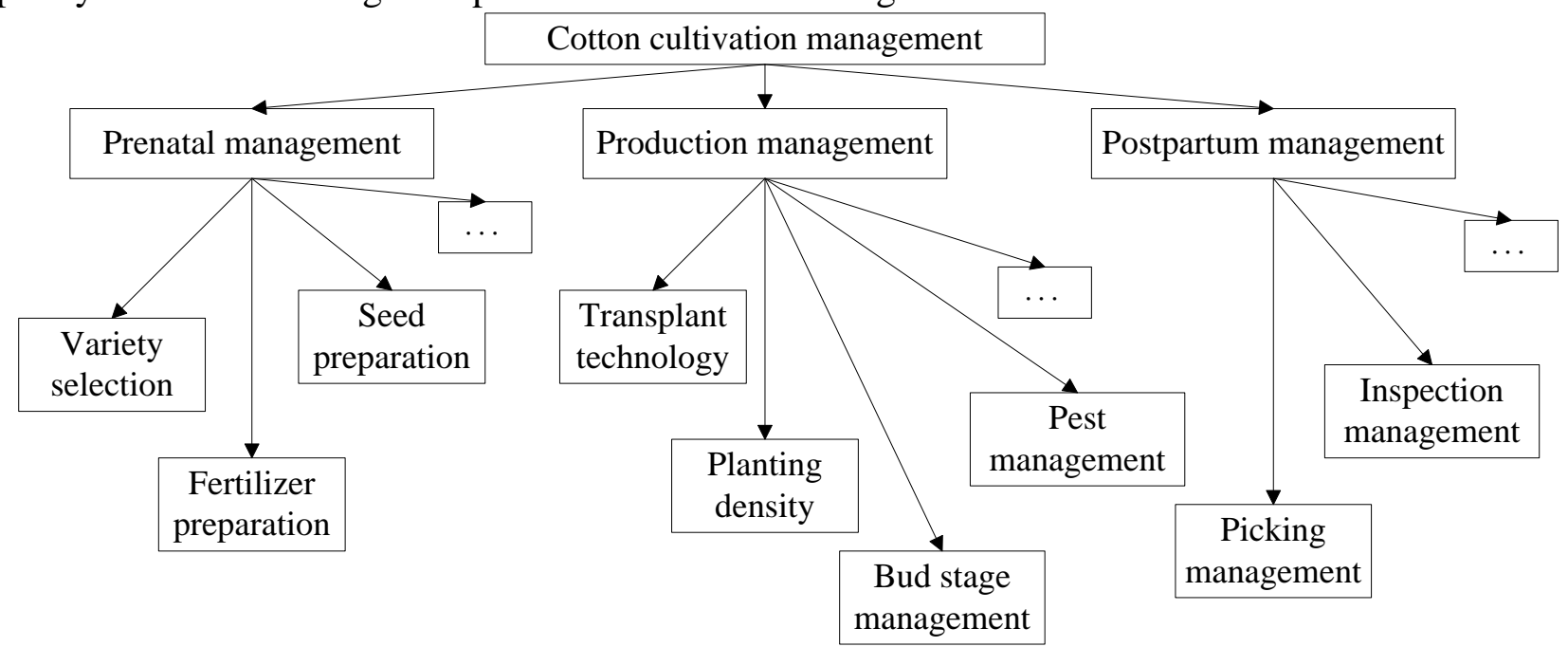

Figure 3. The production rules knowledge-framework 
With the rapid development of computer technology, people-oriented information management has shifted as to the management of the main machine. Most of today's social work is done by community members in a particular group environment through collaboration to jointly accomplished work has obvious distribution, population and collaboration, which requires the ability to interact and collaborate among group members work together to complete the task.

Fig. 4 is a method for predicting the present system of paddy moisture content of the sample and standard deviation values for dry moisture analyzer measured. Measured in 13 samples, sample bias $3,4,5$ absolute value between $1 \%$ to $2.3 \%$, and the remaining samples measuring deviations were less than $0.8 \%$, greater deviation measurement samples 3,4,5 reason Since the actual moisture within the rice grain bulk of these three samples is relatively high, balance and stability required for a long time, the longer the equilibrium predictions stabilization time closer to the true value.

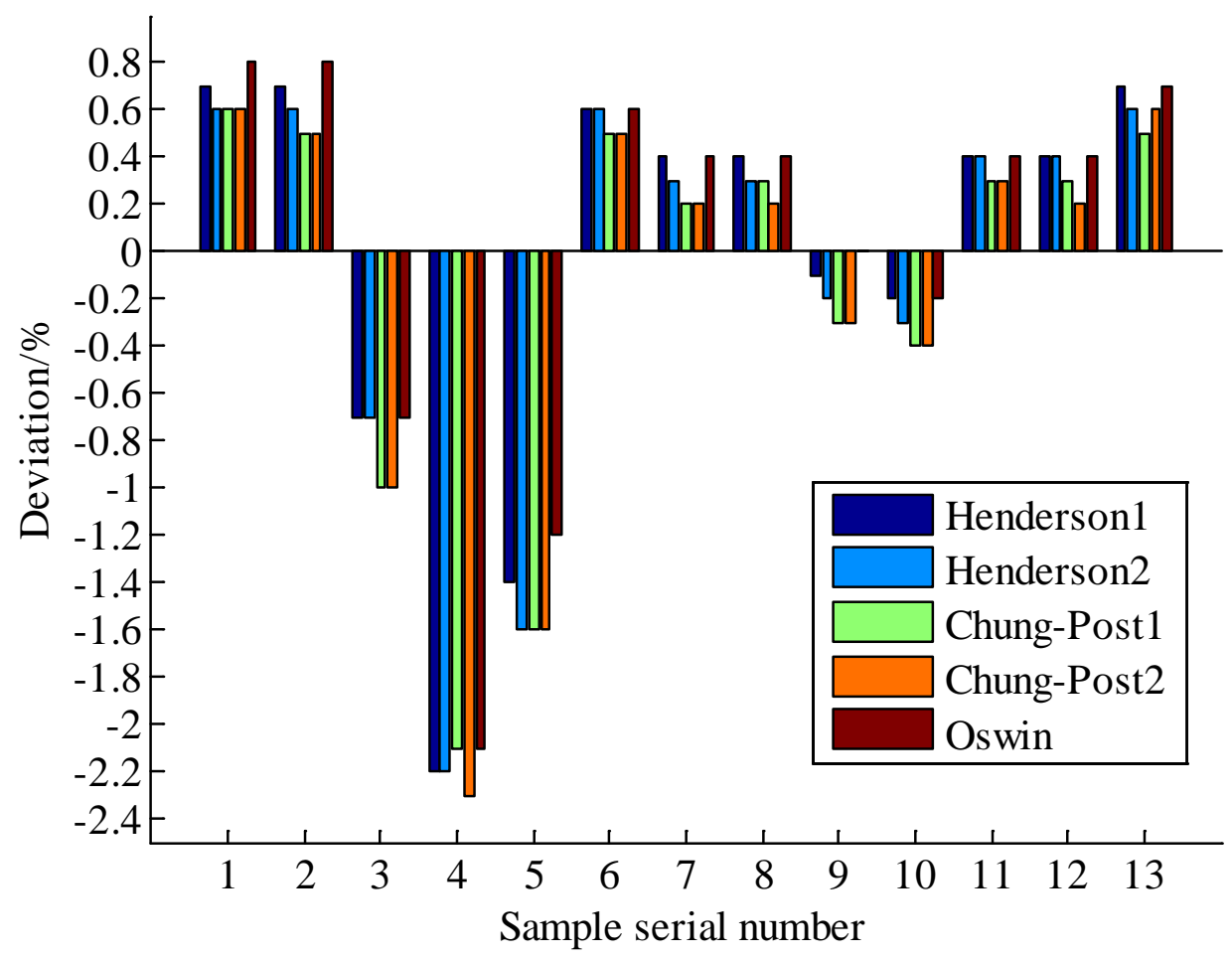

Figure 4.The method for predicting the present system

Dimensional bar code design. Data Matrix two-dimensional bar code is a square or rectangular symbol made up of many small squares composed, store their information is based on permutations and combinations of light and dark squares in two ways byte code encoding, each small square cell is a basic unit. In the encoding, dark on behalf of " 1 ", light stands for " 0 ", the use of clusters of light and dark squares to describe the special character of information, fitted to a complete matrix code according to the encoding rules. The maximum capacity of the Data Matrix two-dimensional bar code characters is 2335, the maximum capacity of 2116 figures.

According grain of origin information traceability rules, using characters to describe grain origin information, and therefore the preparation of information required to meet grain uniqueness, scientific, scalability, stability, simplicity and adaptability requirements. To the source of origin information is encoded as "string AB000105 " to illustrate the encoding process Grain Information Data Matrix two-dimensional bar code. Table 1 ASCn information coding process strings. The coding rule (1)," A ", "B" characters are converted into binary characters "01000010" and "01000011"; according to the encoding rules (3), "00", "01", "05" characters are converted into binary characters "10000010", "10000011" and "10000111. " 
Table 1. Date Matrix encodation schemes of ECC200

\begin{tabular}{lll}
\hline Coding scheme & Character & Bit / data character \\
\hline ASCII & Double digits (decimal) & 4 \\
& ASCII value 0-127 & 8 \\
& ASCII extended value 128-255 & 16 \\
C40 & Basic capital letters digital type & 5.33 \\
Text & Basic lowercase letter type & 5.33 \\
X12 & ANSI X12EDI data set & 5.33 \\
EDIFACT & ASCII value 32-94 & 6 \\
Base 256 & All byte value 0-255 & 8 \\
\hline
\end{tabular}

Reed-Solomon error correction code can correct two types of errors: e error (on a known location can not be scanned or decoded symbol character) and t error (unknown position error is decoded symbol character). Fault tolerance:

$$
e+2 t \leq d-p(1)
$$

Error correction code word is a data code word is error correction code word polynomial $G(x)$ in addition to the remainder obtained namely:

$$
R(x)=\frac{M(x) x^{n-k}}{G(x)}(2)
$$

When measuring readable rates, respectively, from the two-dimensional bar code printing three groups of two kinds of traceable particles randomly 200 samples, each sample scanned three times, if there are 2 or 2 times more readable, it is assumed that the sample can be read, is "1", otherwise is " 0 ." Therefore standard readable percentage rate (Equation 3 ) and readable rate difference (Equation 4) as follows:

$$
\begin{gathered}
p(\%)=\frac{100 * k}{N} \\
S E=\sqrt{\frac{p(1-p)}{N}}
\end{gathered}
$$

Where $\mathrm{k}$ is the scan result is " 1 ", the number of samples, $\mathrm{N}$ is the total number of samples.

\section{Summary}

Things agriculture is one of the eight categories of things divide the most important direction of development. Things existing agricultural research focused on the use of agricultural resources, agro-ecological environment monitoring, agro-ecological fine management, safety of agricultural products traceability, etc., the key technologies in agriculture three-tier model where things are covered, major focus on agriculture perception of information, transfer of agricultural information, agricultural information processing to expand research traitor. Among them, the agricultural information processing technologies include agricultural forecasting warning technology, intelligent control of agricultural technology, agricultural intelligent decision technology, agricultural technology and agricultural visual diagnostic reasoning process technology.

\section{References}

[1] Q. Li, Z.M. Shen, L. wang Gao, H.Y. Yang, Wei Liu, Jian Qiao. An Internet-Based expert system platform for assistant identification of agricultural pests //World Automation Congress (WAC),2010 International Conference on IEEE.2010:141-144.

[2] Rahimi-Vahed A, Mirzaei A H, "A Hybrid Multi-Objective Shuffled Frog-Leaping Algorithm For A Mixed Model Assembly Line Sequencing Problem", Computers and Industrial Engineering, , vol.53,no.4, pp.642-666, 2007. 
[3] C.C.Aggarwal, P.S.Yu. "Data Mining Techniques for Associations.” Clustering and Classification. 2002, pp.22-67.

[4] Anu Vajdyanathan. "Malcolm Shorc and Mark Billingburst.” Data in Social Network Analysis. 2008, pp. 23-25.

[5] Monika Henzinger. "Link Analysis in Web Information Retrieval.” IEEE Data Engineering Bulletin. Sep 2000, pp. 3-8.

[6] M. Sadek, A. Tarighat, A. H. Sayed, “A Leakage-based Precoding Scheme for Downlink multi-user MIMO Channels”, IEEE Transactions on Wireless Communications, vol. 26, no.8, pp.1505-1515, 2008.

[7] Y.Z. Zhu, G.X. Zheng, Y. Rui, , M.Q. Li, "A Novel Distributed Precoding Scheme Based on THP for Downlink Multi-Cell Multi-User OFDMA Wireless Systems", IJACT: International Journal of Advancements in Computing Technology, vol. 5, no. 9, pp. 213-220, 2011.

[8] Raore Soungalo, Li Renfa and Zeng Fanzi, "Evaluating and Improving Wireless Local Area Networks Performance”, IJACT: International Journal of Advancements in Computing Technology, vol. 3, no. 2, pp. 156-164, 2011.

[9] T. Okamoto. A digital multisignature scheme using bijective public-key cryptosystems. ACM Trans. Computer Systems, 6(4), ACM Press, New York, 1988, pp.432-441.

[10] A. Boldyreva. Threshold signature, multisignature and blind signature schemes based on the gap-Diffie-Hellmangroup signature scheme. In Proceedings of PKC 2003, LNCS 2567 , Springer, Berlin, 2003, pp.31-46. 